\title{
Les noces de sang macédoniennes ou comment marier fiction et histoire dans le roman Que demandent les barbares de Dimosthénis Koúrtovik
}

Macedonian Bloody Wedding or How to Match Fiction and History in Dimosthenis Kurtovic's Novel What the Barbarians are Looking For

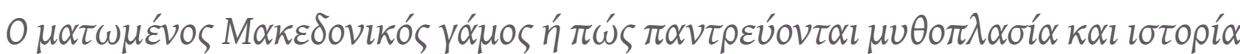

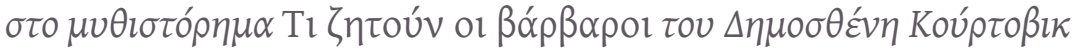

\section{Nicolas Pitsos}

\section{(2) OpenEdition}

\section{Journals}

Édition électronique

URL : https://journals.openedition.org/ceb/8819

DOI : $10.4000 /$ ceb.8819

ISSN : 2261-4184

\section{Éditeur}

INALCO

Édition imprimée

ISBN : 978-2-85831-229-0

ISSN : 0290-7402

Référence électronique

Nicolas Pitsos, « Les noces de sang macédoniennes ou comment marier fiction et histoire dans le roman Que demandent les barbares de Dimosthénis Koúrtovik », Cahiers balkaniques [En ligne], 43 | 2015, mis en ligne le 25 juillet 2017, consulté le 06 juillet 2021. URL : http://journals.openedition.org/ ceb/8819; DOI : https://doi.org/10.4000/ceb.8819

Ce document a été généré automatiquement le 6 juillet 2021

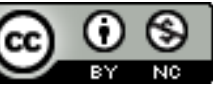

Cahiers balkaniques est mis à disposition selon les termes de la Licence Creative Commons Attribution - Pas d'Utilisation Commerciale 4.0 International. 


\title{
Les noces de sang macédoniennes ou comment marier fiction et histoire dans le roman Que demandent les barbares de Dimosthénis Koúrtovik
}

\author{
Macedonian Bloody Wedding or How to Match Fiction and History in \\ Dimosthenis Kurtovic's Novel What the Barbarians are Looking For

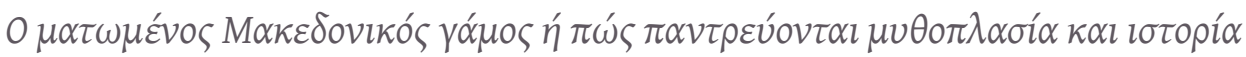

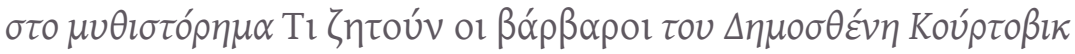

\author{
Nicolas Pitsos
}

1 Dimosthénis Koúrtovik ${ }^{1}$, l'auteur, né à Athènes en 1948, place l'intrigue de son ouvrage Que demandent les Barbares ${ }^{2}$ dans une ville imaginaire, aux frontières entre la Grèce, la République de Macédoine et la Bulgarie. Lors d'un concours interbalkanique pour le prix du meilleur roman, le jury se trouve confronté à un événement exceptionnel et intrigant : trois des ouvrages candidats, bulgare, grec et serbe, traitent du même sujet, un mariage sanglant dans un village macédonien à la veille de la Deuxième Guerre balkanique de $1913^{3}$ alors que sont présentes dans la région trois armées d'occupation, bulgare, grecque et serbe. Les discussions du jury seront par la suite consacrées à la critique de telle ou telle autre version des événements présentée par les romans en question et plus tellement à leur valeur littéraire. L'auteur soulève la question, très intéressante à mon avis, de la place de l'événement historique dans la narration littéraire et des rapports complexes et ambivalents entretenus entre fiction et histoire, dans le cadre d'un roman historique.

2 Les différentes histoires inhérentes à cet ouvrage, se déroulent dans deux lieux, s'articulent autour de deux temporalités, s'inscrivent dans deux contextes historiques macédoniens : d'une part, le présent dans sa version grecque, d'autre part, le passé 
pendant les conflits balkaniques de 1912-1913. Dans cette narration, on retrouve à la fois le regard de l'écrivain sur le passé et des échos du présent retenus et analysés par ce même auteur. Ce qui m'intéresse dans ce roman, en tant que praticien de l'histoire culturelle et disciple des Cultural Studies ${ }^{4}$, c'est aussi bien la représentation d'une certaine époque du passé que la perception de l'actualité proposée par l'écrivain.

Concernant les deux lieux de l'action romanesque, on a d'un côté une petite ville aux frontières entre les trois pays et, d'autre part, le village macédonien indiqué comme $\mathrm{P}$. dans le roman candidat grec, Krousevo ou Kristalli dans le roman candidat serbe, et situé dans les deux cas au sud de la ville de Gevgeli, à la frontière actuelle entre la Grèce et la République de Macédoine.

Enfin, deux événements structurent la narration et tissent la trame romanesque. En premier, le concours littéraire coïncidant avec le centenaire de ce qu'on appelle dans l'historiographie grecque la lutte macédonienne, et qui comme l'affirme le narrateurauteur lui-même, n'est autre qu'une lutte pour la démarcation des sphères d'influence entre les différents nationalismes convoitant la région de la Macédoine ottomane. Le deuxième événement constitutif de la narration est un mariage sanglant de juin 1913.

\section{Entre intertextualité et hypertextualité}

5 La thématique de la noce ensanglantée fait allusion à la pièce homonyme de Vojdan Cernodrinski, Makedonska Krvava Svadba, pièce fondatrice de la dramaturgie macédonienne ${ }^{5}$. L'intrigue de la pièce de Cernodrinski se situe elle aussi dans un village en Macédoine, mais son temps et ses protagonistes ne sont pas les mêmes. Dans cette pièce, le mariage de la jeune Cveta, finit dans un bain de sang par l'intervention des représentants de l'ordre ottoman pendant l'ère d'avant les guerres balkaniques. Plus récemment, un historien grec, Dimitris Lithoxoou, qui a beaucoup travaillé sur la question macédonienne, développant une approche diamétralement opposée à la version de l'historiographie officielle et nationaliste, a publié lui aussi, un article sur un fait historique concernant des noces mises à sang par une bande d'andartès grecs, en $1904^{6}$.

6 L'intertextualité mise à part, le roman de Koúrtovik mobilise également des procédés d'hypertextualité 7 , dans la mesure où les romans candidats au concours Interbalkan sont censés s'être inspirés d'autres textes-sources: le journal intime d'un militaire dans le cas des romans grec et bulgare, et les récits du grand-père du protagoniste dans le roman serbe. Cette dimension hypertextuelle du roman de Koúrtovik est davantage mise en exergue et soulignée dans et par le prologue. Selon l'écrivain, à l'origine de ce roman, il y avait un document cinématographique : un film d'une durée très courte, à peine une minute et demie, tourné par les premiers cinéastes balkaniques, les frères Manakia. C'est grâce à Fotis Lambrinos, spécialiste des rapports entre histoire et cinéma, que l'écrivain aurait appris l'existence de ce film ${ }^{8}$. D'après les informations qu'il aurait obtenues, on y voit un village désert en Macédoine à la veille du deuxième conflit balkanique de 1913. La caméra de ces frères Lumières des Balkans aurait immortalisé la présence de soldats des trois nationalités, bulgare, grecque et serbe, à côté de deux arbres où étaient pendus des gens décapités ou des sacs, et quelque chose comme une fosse dont les soldats ne laissaient personne s'approcher. Il s'agit comme le souligne Marta Cichocka, «de mettre l'accent sur le caractère textuel et narratif, lacunaire et latéral de ces procédés $»^{9}$. En même temps, l'écrivain renie d'emblée son 
statut de romancier et se contente de celui de narrateur. D'autre part, certains historiens, influencés par le discours de Hayden White sur la métahistoire, s'interrogent eux-aussi sur leur statut et la place de l'histoire entre science et fiction. Paul Ricœur mettait déjà en évidence dans son ouvrage Temps et récit les points communs et les trajets parallèles entre l'historiographie et la littérature qui consistent en une présentation par la voie du langage des structures conditionnées par le temps ${ }^{10}$. De son côté, Robin George Collingwood considère que l'œuvre de l'historien et celle de l'écrivain se basent toutes les deux sur un travail d'imagination. Mais alors que l'écrivain a comme mission de construire un tableau cohérent, l'historien doit à la fois composer un tableau qui fait sens et qui représente les événements comme ils se sont réellement passés ${ }^{11}$.

7 Le cadre conceptuel dans lequel j'ai voulu inscrire ma réflexion est celui délimité par les interrogations des théoriciens de la littérature sur la nature du roman historique et des historiens sur l'écriture de l'histoire comme narration fictive. Le roman qui me sert de support et de guide dans cette problématisation est Que demandent les barbares, un roman de la littérature balkanique ou plus précisément un roman sur la littérature balkanique.

8 Mais, qu'est-ce que la littérature balkanique? Pour répondre à cette question, il faudrait plutôt commencer par s'interroger sur la définition des Balkans. Zlatan Zouritch, le représentant de la Serbie-Monténégro du jury, interpellé lui aussi par cette question, propose lors d'un entretien, une image métaphorique très forte. Les Balkans seraient

une grappe de raisins dépourvue de grains (...) la Croatie et la Slovénie ne sont plus là, et maintenant, ces pays soutiennent qu'ils n'ont jamais fait partie des Balkans. Les Roumains vacillent, vous allez voir qu'une fois membres de l'Union européenne, ils ne condescendront plus à participer à ce type de réunions balkaniques... et enfin, vous les Grecs, eh bien, vous étiez les premiers à regarder ailleurs, et depuis un bon moment déjà ${ }^{12}$.

9 Les Balkans sont une invention des géographes européens du XIX ${ }^{e}$ siècle, comme nous l'a très bien montré Maria Todorova ${ }^{13}$. Jusqu'au Congrès de Berlin, les observateurs extérieurs raisonnaient en termes de Turquie d'Europe, d'Empire ottoman européen, de Levant européen, de péninsule orientale, et dans la nomenclature ottomane, on désignait cette région sous le nom de Rumeli-i-chahane [Rumélie impériale], ou Avrupa-i Osmani, c'est-à-dire, Europe ottomane. Les Balkans sont aussi situés au cœur de la question d'Orient dans sa composante ottomane tout au long du xix siècle, et au cœur de la Nouvelle question d'Orient au crépuscule du $\mathrm{xx}^{\mathrm{e}}$ siècle dans sa version yougoslave, avec son cortège d'horreurs, et sa litanie de malheurs ${ }^{14}$. Les Balkans deviennent ainsi un lieu stigmatisé, l'incarnation absolue du Mal, de la sauvagerie, de l'arriération, l'image d'une altérité repoussante et d'une identité accablante que personne ne revendique. Au centre de cette région, la Macédoine résume bien la confrontation entre impérialismes des grandes puissances et nationalismes locaux.

\section{Les conditions du présent}

\section{Les lieux}

Le spectre de la question macédonienne arpente toujours, à l'aube du Xxi siècle, les dédales diplomatiques et sillonne l'espace narratif du roman. L'écrivain évoque le 
différend gréco-macédonien autour de l'appellation de la République de Macédoine. Dans son ouvrage, ce pays est considéré comme " condamné à s'appeler l'ex-République yougoslave de Macédoine $»^{15}$. Ce commentaire ironique de l'auteur met en évidence l'absurdité d'une situation qui persiste depuis une vingtaine d'années, suite au refus de l'État grec de reconnaître l'ex-République yougoslave de Macédoine sous son nom constitutionnel de Macédoine.

11 Le même imbroglio linguistique ou, je dirais, le même palimpseste toponymique, apparaît lorsque l'on discute du nom de la ville où a lieu le concours Interbalkan. Le narrateur nous dit que «les invités Turcs ont poliment remarqué que cette ville s'appelait jadis Tazetepe. Le maire l'admet, tout en justifiant le choix de Tyrimmeia au nom de son ancienneté présumée, rendant hommage au roi de Macédoine Tyrima ${ }^{16}$, encore un personnage fictif, qui aurait fondé la ville aux alentours de 700 avant J.-C. Quant à sa désignation sous le nom de Krasnitsa, le narrateur nous informe qu'elle fut évoquée «lors de rencontres informelles entre certains de nos voisins du Nord, et furtivement, en ville, par les gens du coin $»^{17}$.

On retrouve ces luttes symboliques en vue de l'inscription de la mythologie nationale dans l'espace ${ }^{18}$ avec effacement des traces de la présence de l'Autre, lors d'une visite au village Nitsa près de Tyrimmeia, aux pieds de la montagne du Kaïmaktsalan. C'est le lieu d'où est originaire le maire de Tyrimmeia, Odysseas Traïkos qui affirme que :

les gens croient que le nom du village est issu du diminutif du prénom féminin Eleni, Elenitsa, Nitsa. Cependant, la vérité est toute autre. Cette appellation provient du mot local nitse qui signifie à personne. Les habitants du village n'en pouvaient plus d'être tiraillés tantôt par les occupants bulgares, grecs ou serbes ${ }^{19}$, au moment de la politique imposée de changement de toponymes dans les années 1920, ils ont décidé de proposer ce nom aux autorités grecques, qui ont cru qu'il s'agissait d'un nom entièrement et profondément grec !

On apprend aussi que le nom de famille du maire vient de Traikov. Ici, l'auteur évoque les politiques d'assimilation forcée et d'homogénéisation culturelle mises en place par les nouveaux conquérants et colonisateurs de l'espace macédonien et des Balkans ottomans suite aux guerres balkaniques. Dans un tel contexte, le changement de noms de lieux a été très souvent suivi et doublé d'un changement de noms des gens, les terminaisons de noms à consonance allophone, remplacées par d'autres considérées plus proches à la langue officielle.

\section{Les habitants}

14 Ces politiques, ainsi que les événements historiques qui les ont suivis, ont en grande partie façonné la mosaïque démographique des Balkans et aussi de la Macédoine grecque. L'auteur met en scène les représentants de différentes communautés (autochtones slavophones ou grécophones, descendants des réfugiés, immigrés récents) que les aléas de l'histoire ont réunis dans cet espace. Ce faisant, il s'attaque à l'un des piliers de la politique officielle de l'État grec qui vise à minimiser voire à nier l'existence des communautés ethnolinguistiques autres que grécophones sur son territoire. En même temps - et c'est là, à mon avis, un témoignage précieux pour les praticiens des sciences sociales, historiens ou sociologues - il met en scène des phénomènes et comportements présents dans la société grecque de nos jours, tels que le racisme ou la xénophobie. 

récemment arrivés. Lors d'un spectacle organisé par le sponsor de l'événement, l'entrepreneur local Aris Xinos ${ }^{20}$, des syndicalistes grévistes font intrusion sur la scène en criant des slogans contre les licenciements, la délocalisation de l'industrie viticultrice en Bulgarie et contre les Albanais comparés à des rats. Si les pratiques d'une économie néo-libérale complètement déréglementée et entièrement dérégulée sont évoquées, les discours racistes énoncés par des ouvriers en quête de boucs émissaires en période de crise sont également épinglés et exhibés. En même temps, le phénomène de l'exploitation des ouvriers immigrés est ouvertement exposé, quand Cyrille Stoyanov s'adressant à Argyris, commente la sociologie de la boîte de nuit où ils se trouvent: «La serveuse, si j'ai bien compris, doit être roumaine (affirme-t-il). Ceux qui sont à la vaisselle dans la cuisine doivent être albanais. Après minuit, des stripteaseuses Russes, Ukrainiennes et Moldaves viendront danser sur la piste et une fois leur spectacle fini, elles seront obligées d'ouvrir leurs cuisses aux clients demandeurs. Tout ce personnel international, des gens diplômés, s'épuise et s'humilie afin que son directeur, très probablement un Grec semi-analphabète, puisse s'enrichir $»^{21}$.

À plusieurs reprises dans le roman, l'auteur-narrateur évoque le phénomène des nouveaux riches avec leurs Land Rover, leurs villas avec gazon, qui se sont enrichis grâce à l'exploitation des individus en détresse arrivés en Grèce suite à l'effondrement des régimes autoritaires et totalitaires dans l'Europe de l'Est.

À côté de ces immigrés, figurent également dans le roman, les descendants des réfugiés, comme Xinos lui-même, dont le grand-père était originaire de Plovdiv, de Philippopoli. L'auteur évoque à cette occasion, un des nombreux exemples de déplacements de populations dans les Balkans, lors de l'annexion de la Roumélie orientale à la Bulgarie en 1885. Son héros, tout en déclarant que la mémoire traverse le temps et déborde les frontières d'une génération, s'affirme exempt de toute rancœur. Il déplore seulement la perte de la diversité culturelle qui faisait la richesse de l'espace balkanique, grâce à la coexistence au sein d'une même entité politique, de diverses communautés culturelles.

Les Makedonski font partie de cette diversité ; dans la Macédoine grecque, il s'agit des populations locales de cette région, de langue macédonienne. À leur propos, Argyris, le président du jury, interroge le chauffeur de taxi qui l'amène dans la ville où aura lieu le concours littéraire. Celui-ci lui répond que

nous n'en voulons plus à ceux-là. Depuis qu'on les laisse chanter leurs chansons, danser leurs danses, ils se sont calmés. Dans le temps, les jeunes parlaient leur parler local, et chantaient leurs propres chansons par caprice (...) maintenant ils se tournent de plus en plus vers les chansons grecques, la musique populaire (...) et les chansons étrangères, surtout les chansons américaines ${ }^{22}$.

19 Afin de mieux comprendre la réalité sociopolitique à laquelle l'auteur fait allusion dans cet extrait, il faut savoir que l'État grec a été à plusieurs reprises condamné par la Cour européenne des droits de l'homme, au sujet de sa politique quant aux droits des minorités ${ }^{23}$. Au sujet des Makedonski, l'auteur soulève aussi la question très délicate, mais essentielle pour la compréhension de la construction des identités balkaniques, du sens de la nation, dans une région où cette notion a été importée et imposée à des gens qui ne concevaient pas du tout leur existence en ces termes.

20 Le discours de Trifonas, le propriétaire de la taverne dans le village de Nitsa, est révélateur de la confusion mentale et sémantique s'exprimant toujours à propos de 
l'emploi de ce concept. Un jour s'adressant à un journaliste de la République de Macédoine, il lui annonce que les réfugiés d'Asie Mineure, installés dans le village, avaient occupé les maisons de nos sanarodnitsi. Le journaliste déduit de cette remarque que Trifonas le considère comme son " omoethnis », c'est-à-dire celui qui appartient à la même nation. Or, Trifonas avait employé le mot dans son sens tribal et clanique d'«omogenis", car lui, il se considère comme Macédonien avec une conscience nationale grecque. Le terme genos est plus proche à mon avis de l'organisation sociale de l'Empire ottoman en millet, alors que le mot ethnos a été utilisé pour rendre le terme de nation, sans pour autant en être synonyme et gardant toute son ambivalence sémantique avec ses références constantes à sa dimension ethnique qui le rapprocherait d'une définition allemande de la nation en termes de Volk, plutôt qu'à une définition française en termes de plébiscite quotidien ${ }^{24}$ et par conséquent appartenance consentie et consciente et non pas subie et inconsciente.

\section{Les fantômes du passé}

\section{Les mémoires d'Argyris}

21 À côté des êtres vivants, il y a aussi dans ce roman, les fantômes du passé qui ressurgissent dans la mémoire des protagonistes. Le président du jury se souvient de la Macédoine de son enfance, de «ce pays de la magie amputée et des mystères profanés $»^{25}$. Des images d'atrocités de l'Occupation bulgare pendant la Seconde Guerre mondiale, de la guerre civile, des images désolantes des exécutions de détenus politiques à la prison de Salonique surnommée les Sept Tours (Eptapyrgio, en grec, Yedi kulé, en turc) côtoient les images « des ombres noires planant, errant là où gisait jadis le cimetière juif, avec les tombes creusées et les pierres tombales brisées et les sons ahurissants et sidérants des coups de pioche sourds et des murmures se terminant dans un juron grec sonore, au nom de la peine perdue ${ }^{26}$.

À côté de ce tableau funèbre et macabre ${ }^{27}$, un environnement sonore résonnant d'une pluralité linguistique refait surface dans la mémoire d'Argyris. Il s'agit d'une polyphonie qui n'était pas enseignée dans les manuels scolaires de l'endoctrinement patriotique, mais qui a été au contraire muselée, réprimée et persécutée.

Parmi les intonations qui jaillissent des sources souterraines de son subconscient, lors de cette immersion proustienne, il y a "les mots grecs accentués à la pénultième syllabe par les autochtones grécophones ${ }^{28}$, les «mots slavomacédoniens des autochtones slavophones, échangés à la dérobée $»^{29}$ allusion aux politiques de répression linguistique appliquées par les régimes autoritaires en Grèce, tels que la dictature de Metaxás, le grec des réfugiés d'Asie Mineure avec sa syntaxe turque, le karamanli des habitants de Karaman, le pontique d'autres groupes de réfugiés venant de Turquie ${ }^{30}$ et désignés parfois comme Tourkomerites « ceux venant de Turquie », terme synonyme pendant longtemps de stigmatisation, discrimination et marginalisation de ces populations dans l'espace public, «le parler dégringolant des Tsiganes $»^{31}$, «le sépharadite d'un air un peu miaulant et chantant $\star^{32}$ des juifs de Salonique décimés pendant l'Occupation allemande, voire même les expressions de son père qui à l'occasion des moments de détente familiale «laissait s'échapper des mots valaques, c'était alors qu'il appelait manià sa grand-mère $»^{33}$. 

représentant bulgare du jury et son arrière-grand-père, partisan du VMRO, l'organisation révolutionnaire macédonienne se battant pour la création d'une Macédoine indépendante, organisée sur la base d'une société fédérale respectueuse de son caractère multiculturel et multiconfessionnel. Le vieux Stoyan était assis sur le seuil de la vieille église de Saint-Isidore, les mains posées sur ses genoux, quand tout d'un coup son arrière-petit-fils s'approche de lui.

Qu'est-ce qui t'amène ici mon fils? Je suis venu pour un prix, papy, qui traite des guerres balkaniques, de la Macédoine. Macédoine, Terre esclave, se mit à chanter le vieux Stoyan (...) Toi, tu es Bulgare (...) moi, je n'ai jamais eu ce sentiment (...) Je ne comprends pas ce que veulent dire les États, les Nations (...) je connais seulement le souffle de ma terre. Ma patrie, c'est la Macédoine, la terre où sont enfouis les os de mes grands-parents. La Macédoine a connu plusieurs esclavages. Les États que vous avez formés en ont rajouté. Tous les États sans aucune exception ${ }^{41}$. 


\section{La discussion entre Anton et Fotis}

29 Anton et Fotis, deux soldats morts, tombés sur les champs de bataille lors de la Deuxième Guerre balkanique, l'un du côté grec, l'autre du côté bulgare, sont des victimes de ces opérations de nationalisation de la région de Macédoine au début du $\mathrm{xx}^{\mathrm{e}}$ siècle. Leurs ombres parcourent le salon où se tient le concours littéraire. Lors d'une séance pour la sélection du roman qui recevra le prix Interbalkan, ils assistent à une querelle, à une joute littéraire sur le terrain de l'histoire. L'histoire mobilisée à l'époque des guerres balkaniques pour légitimer telle ou telle autre revendication nationale-nationaliste, intervient de nouveau comme juge suprême de la valeur littéraire d'un roman.

\section{Les noces macédoniennes ensanglantées entre histoire et fiction}

Dans le roman de Koúrtovik les relations/frontières entre fiction et histoire sont longuement débattues à l'occasion des commentaires faits par les protagonistes de la trame. Nina Daneva, l'écrivaine bulgare dans le roman, raconte que c'est par l'intermédiaire de son arrière-grand-mère qu'elle a reçu le journal intime de Dimtcho Débélianov (1887-1916), illustre représentant de la poésie symbolique en Bulgarie et personnage bien réel. En feuilletant ce journal, Daneva serait tombée sur l'événement des noces sanglantes. Suite à cette confession-révélation, Argyris lui demande si elle avoue avoir raconté une histoire vraie? Et Daneva lui répond: "J’ai écrit un roman. Je n'ai pas copié une chronique $»^{42}$.

31 Un document historique peut être source d'inspiration littéraire. L'écrivain se l'approprie et l'intègre dans un univers fictif. Il ne cherche pas à trouver, démontrer la vérité historique, au contraire, il propose sa propre version romancée. Par contre, on peut toujours lire un roman comme un effort de manipulation de l'histoire à des fins politiques, ou faire abstraction des vraisemblances historiques et se délecter de sa trame narrative et des mécanismes de sa construction.

32 Ce dilemme majeur, face à la réception/production d'un roman historique, est également commenté dans le roman de Koúrtovik, lors de l'intervention de Jasmina Tzelilbasitch, représentante de Bosnie-Herzégovine dans le jury. Elle remarque au sujet du roman grec que, malgré l'intention affichée de l'auteur, désireux d'orienter son récit vers un dépassement des passions nationalistes et soucieux de lui accorder une dimension réconciliatrice, le résultat est plutôt décevant. L'écrivain semble être toujours négativement prédisposé à l'égard des Bulgares et des Serbes. À cette remarque, Argyris, rétorque qu'un roman n'est pas censé faire de la propagande et que les dénouements moraux devraient être destinés et réservés aux manuels scolaires.

Koúrtovik met le doigt sur un point très délicat dans les relations entre fiction et histoire en nous confrontant, selon moi, à deux questions essentielles : est-on prêt, en tant que lecteur-lectrice à accorder son autonomie à une œuvre littéraire, ou au contraire souhaite-t-on la soumettre à l'épreuve de la rigueur historique? Et puis, quelles sont les intentions de l'auteur en écrivant un roman historique ? Proposer une version romancée de l'historiographie officielle, ou prendre ses distances en la revisitant et en donnant la parole aux opprimés, aux vaincus, aux oubliés, aux occultés 
de cette histoire ? C'est ce que Koúrtovik essaye de faire dans son roman en inventant cette coïncidence des trois romans candidats pour le prix Interbalkan, traitant sous des points de vue différents, du même événement, les noces sanglantes macédoniennes. Trois officiers, un Bulgare, un Grec et un Serbe, arrivent dans un village macédonien, afin d'assister à un mariage en tant que témoins. L'issue de ce mariage est tragique dans les trois versions romanesques. La jeune mariée, native de la province de la Macédoine ottomane, est convoitée par les officiers des trois armées d'occupation qui assistent à la cérémonie. La perte de son futur mari lors des événements sanglants qui marqueront la fin de cette cérémonie, pourrait être lue comme une allégorie commentant le partage de la Macédoine au lendemain de la Deuxième Guerre balkanique. L'aboutissement tragique de la cérémonie nuptiale mis à part, les trois romans présentent des versions divergentes de la trame. Dans le roman grec, intitulé En attendant les Bulgares, la présence des soldats bulgares est décrite, dès le départ, comme massive et menaçante. Dans le roman bulgare, intitulé le Deuil du cerisier, l'officier bulgare est présenté comme un cousin de la jeune mariée et sa présence au mariage n'est pas considérée comme une intrusion. Dans le roman serbe enfin, intitulé le Dictionnaire de l'Au-delà, le pont du village où a lieu le mariage est finalement sauvé et n'explose pas. En revanche, dans les variantes narratives grecque et bulgare, ce pont est bombardé. L'identité de l'avion à l'origine de ce bombardement devient un enjeu de taille dans le déroulement du concours. Dans la version grecque, l'écrivain avance l'identité bulgare de l'avion, dans la version bulgare, il s'agirait plutôt d'un avion français et le côté grec se verrait indirectement incriminé car les Français avaient encadré les aviateurs grecs pendant les guerres balkaniques. Le débat sur l'élucidation de ce fait prend une telle ampleur que le président du jury fait appel à un spécialiste des questions militaires, un général retraité, qu'il invite à communiquer son point de vue sur cette épineuse question.

\section{L'écrivain comme gérant de l'histoire}

34 Stoyanof, l'écrivain bulgare du jury, déclare lui aussi, avoir étudié l'histoire militaire et affirme que les forces armées bulgares ne disposaient pas d'avion de modèle Farman, pendant les guerres balkaniques, comme l'écrivain grec semble le suggérer dans son roman. Il conclut donc à une double éventualité : soit le document qui servit de base pour l'écriture de ce roman était dès le départ un faux, soit l'auteur du roman l'a délibérément faussé afin d'attribuer la responsabilité pour le bombardement du pont aux Bulgares.

Pourquoi attacher une si grande importance à cet incident? se demande Argyris. Et son ami Stoyanov lui répond: " tu sais bien que les écrivains gèrent l'Histoire $»^{43}$. Cette réplique sonne comme une révélation pour Argyris qui se rend tout d'un coup compte que le concours qu'il préside, n'est pas une manifestation visant à s'émanciper du fardeau de l'Histoire à travers la littérature ou à s'affranchir des mythologies nationales autour de l'Histoire. Il s'agit plutôt de l'image que le reste de l'Europe voulait avoir de cette contrée si malfamée, de son territoire. Et dans cette perspective, ce n'est pas Argyris qui est le juge, mais c'est bien la journaliste de la chaîne ARTE qui couvre l'événement, représentante de l'Europe, et dont les employeurs ont proposé une adaptation cinématographique du roman qui recevra le premier prix. Cette même journaliste, Outa Raoutenberg, s'adressant aux membres du jury, déclare d'un air plutôt méprisant ne pas vouloir s'immiscer dans leur travail. Pourtant elle les prévient d'une chose : 
si vous désirez vraiment que le prix que vous allez décerner ait un écho quelconque, vous devriez choisir un ouvrage " couleur locale ». J'entends par là, quelque chose qui puisse être internationalement reconnu comme une œuvre issue de cet espace culturel et qui puisse émouvoir par son authenticité indigène. Vous n'irez pas loin avec des allégories postmodernes, des romans-essais, des réflexions sur l'Histoire, parce qu'il y a d'autres écrivains venant d'ailleurs qui réussissent peut-être mieux que vous dans ces domaines. Et il va de soi que vous ne serez pas confortés dans votre démarche en attisant des événements historiques que personne ne connaît et qui n'intéressent que vous ${ }^{44}$.

Il s'agit d'une vision teintée d'une nuance néo-colonialiste, exprimée de la part d'une représentante de l'Europe extra-balkanique, et plus précisément d'un pays, l'Allemagne, dont les visées impérialistes dans les Balkans ont été une des constantes de sa politique extérieure et cela depuis le temps de Drang nach Osten. Il s'agit en même temps, d'une approche réductrice, caricaturale de la littérature balkanique. Les écrivains balkaniques sont considérés comme réfractaires aux innovations littéraires, leurs œuvres intrinsèquement et irrévocablement identifiées et jugées à l'aune d'un exotisme. Je pense que Koúrtovik soulève ainsi la question de la visibilité et de la représentativité de la littérature balkanique au-delà des frontières des pays producteurs, en formulant des hypothèses très pertinentes, me semble-t-il, quant à sa lisibilité restreinte dans le reste du continent européen ${ }^{45}$. À côté des facteurs économiques et politiques redevables de l'organisation et la mise en place des dispositifs adéquats et performants pour la diffusion du livre balkanique, il y aurait aussi des facteurs culturels, tributaires de la représentation du Balkanique et des Balkans. Ce balkanisme littéraire, pour appliquer le terme forgé par Maria Todorova, dans le domaine de l'écriture, est accompagné d'une incompréhension de l'histoire balkanique. Dans un autre extrait du roman, Outa qualifie le rapport entre les écrivains balkaniques et le passé historique de la région, de psychotique.

\section{L'historien comme praticien de la fiction}

Si l'incident de l'avion suscite une telle polémique auprès des observateurs balkaniques et exo-balkaniques, c'est parce que les guerres balkaniques restent des événements insuffisamment abordés dans l'historiographie européenne et diversement assimilés dans les historiographies nationales des pays directement impliqués. En général, deux écoles se distinguent quant à la nature de la Première Guerre balkanique, opposant les historiens qui y voient des opérations de libération à ceux qui les considèrent comme des conquêtes impérialistes et des opérations colonialistes suite à des revendications irrédentistes. Quant à la deuxième guerre balkanique, une des questions les plus épineuses reste la responsabilité du déclenchement des hostilités.

Dans le roman serbe, conçu comme le Dictionnaire des Khazars de Milorad Pavitch, l'écrivain se sert des récits familiaux pour raconter les événements des noces sanglantes dans leur version proposée par Zarko Miloutinovitch, arrière-grand-père de l'auteur, un des premiers aviateurs balkaniques dont l'avion s'écrasa près du village où le mariage a été célébré. Il fut donc témoin oculaire de ce qui s'est passé, et son témoignage, nous dit l'auteur, est complété par ce que lui a raconté son codétenu au camp de Gerlitz pendant la Première Guerre mondiale, un officier grec, Iordanis Latinis, dont le nom est d'une similitude intrigante avec celui de Prodromos Litinas, auteur présumé de la version grecque des Noces de sang dans le roman grec. L'écrivain serbe précise que, puisqu'ils communiquaient dans un italien approximatif, il se peut qu'il y 
ait eu des malentendus. Ici, Demosthenis Koúrtovik, relève à mon avis, deux points majeurs et sensibles pour l'écriture de l'histoire: le rôle des différentes temporalités dans la reconstitution de l'événement historique et la place des médiateurs dans ce processus. Selon Arlette Farge, l'événement se reconstruit sans arrêt et prend de multiples inflexions selon l'époque où il sera reçu. Par ailleurs, la mémoire de l'événement par ceux qui l'ont vécu, intervient dans cette démarche de reconstitution et détermine son sens au fur et à mesure qu'il est revisitée ${ }^{46}$.

Cet événement retracé dans des archives personnelles (journal intime ou entretien) ou officielles (rapports consulaires) est très souvent le résultat de différentes strates de médiation, barrières linguistiques, considérations personnelles, orientations idéologiques. Comment donc, pour l'historien, reconstituer la vérité historique dans un tel contexte et par rapport à des incidents, dont les acteurs et informateurs avancent des versions radicalement divergentes? Devant une telle aporie épistémologique, les événements de l'histoire sont alors vus comme une construction humaine et narrative, laquelle a beaucoup en commun avec la fiction, comme l'a déjà commenté Hayden White dans son ouvrage sur la métahistoire ${ }^{47}$.

Les paroles d'Anton, l'un des deux soldats décédés lors des batailles de la Deuxième Guerre balkanique dans le roman, prolongent ces réflexions théoriques, quand il affirme qu'aucun être vivant ne peut se souvenir d'eux. Pourtant il peut raconter: « Non pas notre vie, mais quelque chose qu'on n'a pas vécu et qui est considéré comme avoir été notre époque $»^{48}$. Cette pensée renvoie aux réflexions de Franklin Ankersmit ${ }^{49}$ sur la logique narrative de l'historiographie, nous invitant à penser les relations entre le processus de narration employé par les romanciers et les historiens.

\section{Conclusion}

41 Le roman de Koúrtovik, Que demandent les barbares, nous offre, une démonstration éloquente de la distinction des romans historiques en deux catégories ${ }^{50}$. D'une part, le roman historique, qualifié d'illusionniste, né de la volonté de l'auteur de créer une illusion d'authenticité et de véracité dans son texte: dans le roman de Koúrtovik, il s'agit des trois candidatures au prix du jury. D'autre part, le roman historique antiillusionniste correspondant à une approche critique de l'histoire : c'est le cas du roman de Koúrtovik dans son ensemble. Le romancier insère les éléments historiques dans un univers fictif tout en attirant l'attention du lecteur sur la discontinuité et l'hétérogénéité $d u$ récit. Son récit ressemble à une mosaïque. Au lieu de présenter l'histoire, il présente des histoires au pluriel. La pluralité des points de vue relatés remet en question la prétention d'une seule vérité historique et permet de confronter diverses interprétations des faits historiques.

En cela, je trouve que le roman de Koúrtovik est fortement influencé par la nouvelle histoire, c'est-à-dire une histoire caractérisée par un nouvel outillage conceptuel, des méthodes novatrices et des sources originales, qui dans le sillage de l'École des Annales et influencée par les Cultural Studies, privilégie la micro-histoire, l'histoire quotidienne, l'histoire des représentations. Argyris méditant sur le roman de Nina, pense que «peu importe finalement qu'elle ait modifié les événements réels. La vraie vérité historique est composée des petites histoires personnelles des êtres humains, éternellement reproduites et pourtant toujours différentes qui tissent le fil du temps $»^{51}$. Cette nouvelle histoire se distingue aussi par une nouvelle éthique. S'opposant aux 
narrations nationalistes dans lesquelles la victime est toujours Nous et le bourreau l'Autre, elle se veut critique face à une héroïsation, sublimation, victimisation du Soi, et une stigmatisation, diabolisation, et dépréciation systématique de l'Autre. Face à une histoire officielle-étatique, qui glorifie et rend légitime toute opération décidée par l'État-nation, cette nouvelle histoire qui a aussi profondément influencé l'écriture littéraire, propose une narration alternative, objective visant à développer une lecture panoramique et empathique du passé, en vue de sa compréhension plus globale et moins partisane, uni-dimensionnelle, instrumentalisée et exclusiviste.

\section{BIBLIOGRAPHIE}

ANKERSMIT Franklin Rudolf, 1983, Narrative Logic: A Semantic Analysis of the Historian's Language, Boston-London-The Hague: M. Nijhoff.

СІСНоскА Marta, 2007, Entre la nouvelle histoire et le nouveau roman historique : réinventions, relectures, écritures, Paris : L’Harmattan.

Collingwood Robin, 1970, The Idea of History, [1st ed. 1946], Oxford: Clarendon Press.

DARQUES Régis, 2002, Salonique au XX $X^{e}$ siècle : de la cité ottomane à la métropole grecque, Paris : CNRS, «Patrimoine de la Méditerranée».

DÉRENS Jean-Arnault, 2006, Batailles d'histoire dans les Balkans, Arcueil : Le Courrier des Balkans. DUKOVSKI Dejan, 2006, Baril de poudre, Balkan's not dead, l'Autre Côté, Paris : L'Espace d'un instant, trad. Frosa Bouchereau.

FARGE Arlette, 2002, « Penser et définir l'événement en histoire : approche des situations et des acteurs sociaux », Terrain, mars 2002, n 38, p. 69-78.

GINZBURG Carlo, 2003, Rapports de force, histoire, rhétorique, preuve, Paris : Gallimard.

KARAKASIDOU Anastasia, 1997, Fields of Wheat, Hills of Blood: Passages to NationHood in Greek Macedonia, 1870-1990, Chicago: The University of Chicago press.

KENNAN George F., 1993, The Other Balkan Wars: a 1913 Carnegie Endowment Inquiry in Retrospect, Washington D.C.: Carnegie Endowment for International Peace, Brookings Institution Publications.

KōSTóPOULOS Tásos, 2007, Guerre et nettoyage ethnique : l'aspect oublié d'une décennie d'expédition nationale, 1912-1922, Athènes : Vivliorama.

KOÚRTOVIK Dimosthénis, 1994, Poussière d'étoiles, Paris : Hatier, trad. Jasmine Pipart.

KOÚRTOVIK Dimosthénis, 2004, la Nostalgie des dragons, Arles : Actes Sud, trad. Caroline Nicolas.

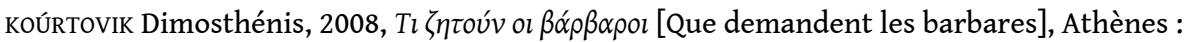
Ellinika Grammata.

KOÚRTOVIK Dimosthénis, 2005, le Hangover grec : essais, Athènes : Nefeli. 
MCDOUGALl Gay, 2008, “Mission to Greece (8-16 September 2008)” (Sur l'implémentation des dispositions prévues dans la Déclaration des droits des personnes appartenant à des minorités nationales, ethniques, religieuses ou linguistiques. Mission de Gay McDougall, expert indépendant de l'ONU). Le rapport rédigé suite à cette mission est disponible à l'adresse http://www.refworld.org/docid/49b7b2e52.html.

MATTELART Armand, NEVEU Érik, 2003, Introduction aux Cultural Studies, Paris : La Découverte, « Repères ».

MAZOWER Mark, 2004, Salonica, City of Ghosts: Christians, Muslims and Jews, 1430-1950, London: Harper Collins.

POIRRIER Philippe, 2004, les Enjeux de l'histoire culturelle, Paris : Éditions du Seuil.

THE FREE AND DEMOCRATIC BULGARIA FOUNDATION, 1996, "The Carnegie Report and the Balkans Today: International Conference on the Balkans", Sofia, March 31-April 2 1995, Sofia: RVR.

TODOROVA Maria, 1997, Imagining the Balkans, New York, Oxford: Oxford University Press.

WEIBEL Ernest, 2002, Histoire et géopolitique des Balkans de 1800 à nos jours, Paris : Ellipses, Collection «L'Orient politique».

WHITE Hayden, 1973, Metahistory: the Historical Imagination in 19th Century Europe, Baltimore, London: Johns Hopkins University Press.

\section{NOTES}

1. Auteur de plusieurs romans, dont Poussière d'étoiles, traduit par Jasmine Pipart et publié aux éditions Hatier en 1994, et la Nostalgie des dragons traduit par Caroline Nicolas et paru aux éditions Actes Sud en 2004. Dimosthenis Koúrtovik travaille également comme critique littéraire.

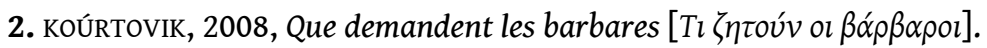

3. Pour une histoire événementielle de ce conflit militaire, voir Ernest WEIBEL, 2002, Histoire et géopolitique des Balkans de 800 à nos jours, surtout le chapitre «Les guerres balkaniques », p. 211-323.

4. Pour une présentation panoramique des Cultural Studies, voir Armand MATTELART, Érik NEVEU, 2003, Introduction aux Cultural Studies, et pour une synthèse des travaux dans le domaine de l'histoire culturelle, voir Philippe POIRRIER, 2004, les Enjeux de l'histoire culturelle.

5. Dejan Dukovski s'en est inspiré pour créer sa pièce Balkans' not dead. Né en 1969 à Skopje, Dukovski, est le principal représentant de la nouvelle génération du théâtre et du cinéma macédoniens, connu surtout pour son scénario de Baril de poudre, traduit du macédonien par Frosa Pejoska et paru dans un recueil réunissant trois de ses pièces traduites en français, DUкоvsкI, 2006, Baril de poudre, Balkans' not dead, l'Autre Côté.

6. Cet article a été publié dans la revue НОВА 3ОРА, 1998, vol. 3, p. 34-35. L'héroïsation et l'idéalisation de cette période de l'histoire balkanique laissent place à une approche critique, révélant les aspects tragiques et peu glorifiants pour les acteurs et pays impliqués dans des confrontations militaires et paramilitaires pour le contrôle de la province ottomane de la Macédoine. 
7. Voir Marta сісноска, 2007, Entre la nouvelle histoire et le nouveau roman historique: réinventions, relectures, écritures. L'auteur affirme que le roman historique peut se considérer comme un genre hypertextuel, dans la mesure où il élabore une histoire déjà racontée par d'autres textes (chroniques, manuscrits, documents, légendes, récits historiques) auxquels il renvoie d'une manière plus ou moins explicite.

8. La dimension hypertextuelle du roman se manifeste dans ce prologue. Comme le souligne Cichocka, cette hypertextualité aurait aussi pu s'exprimer dans un épilogue ou à l'occasion des commentaires métanarratifs, où l'auteur-narrateur s'interroge sur la fiabilité des sources, nuance ou corrige certaines données, le tout pour renforcer l'effet de vraisemblance de l'histoire racontée. Voir сісноскА, Entre la nouvelle..., p. 180.

9. сісноска, Entre la nouvelle..., p. 181.

10. сісноскА, Entre la nouvelle..., p. 96.

11. Voir Robin Collingwood, 1970, The Idea of History, p. 246. S'opposant à ces approches et lectures de l'écriture historienne, Carlo GINZBURG leur reproche leur relativisme envers tout récit historique. Il a réuni ses critiques dans un ouvrage intitulé Rapports de force, histoire, rhétorique, preuve, 2003. Dans celui-ci, il s'élève contre la réduction simpliste de l'historiographie à la rhétorique, opérée par les représentants du linguistic turn, notamment Roland Barthes et Hayden White. Selon lui, cette démarche favorise une argumentation antipositiviste, profondément sceptique quant à l'objectivité de l'histoire.

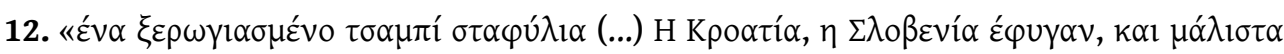

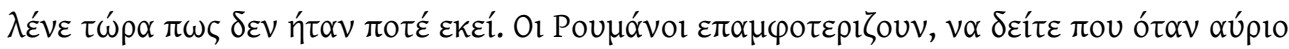

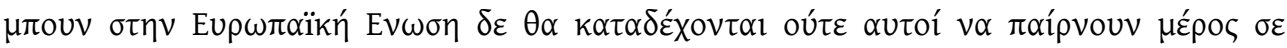

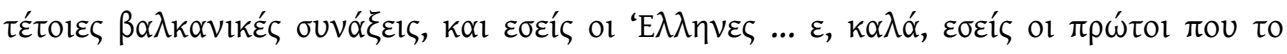

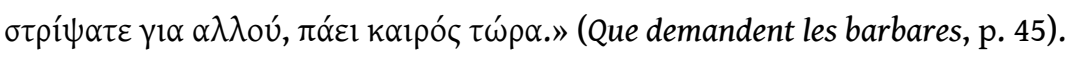

13. Voir Maria TODOROVA, 1997, Imagining the Balkans, surtout le chapitre "The Balkans: nomen", p. 21-37.

14. Pour une étude comparative qui dégage les similitudes entre les guerres balkaniques de 1912-1913 et celles des années 1990, voir les comptes-rendus d'un colloque international sur les Balkans organisé par The Free and Democratic Bulgaria Foundation, 1996, "The Carnegie Report and the Balkans Today: International Conference on the Balkans", et surtout l'intervention d'Oya Akgonenc Mughisuddin qui retrace les similitudes entre les deux périodes de conflit (p.81-85). L'auteur dénonce des pratiques de nettoyage ethnique prônées et soutenues par un fanatisme religieux, communes dans les deux périodes historiques.

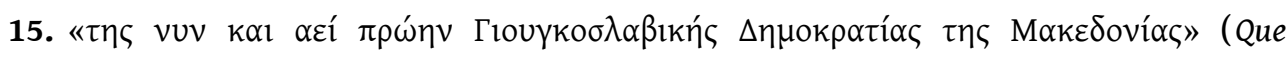
demandent les barbares, p. 16).

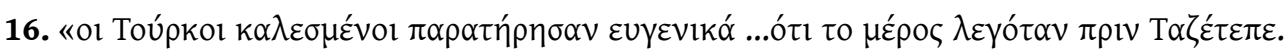

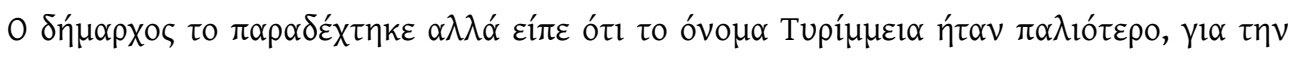

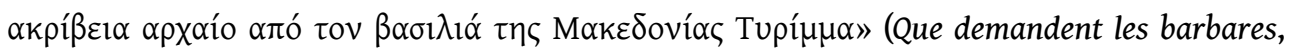
p. 16).

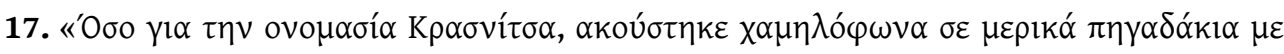

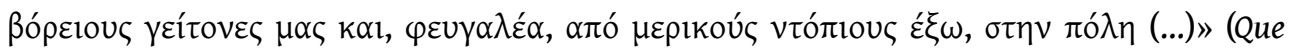
demandent les barbares, p. 16). Sur la question des différents peuplements de la région de 
la Macédoine en Grèce, voir l'étude d'Anastasia KARAKASIDOU, 1997, Fields of Wheat, Hills of Blood: Passages to NationHood in Greek Macedonia, 1870-1990.

18. Sur la question d'élaboration de mythologies nationales par les États balkaniques, voir Jean-Arnault DERENS, 2006, Batailles d'histoire dans les Balkans. L'auteur y évoque l'obsession des origines et l'idéologème de continuité historique comme éléments constitutifs et piliers conceptuels de ces mythologies.

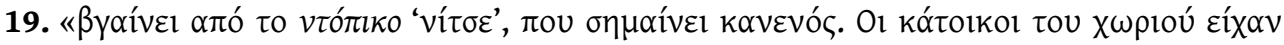

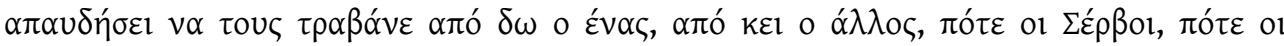

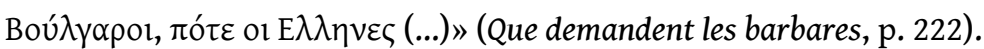

20. Désignation ironique de ce personnage viticulteur de métier et dont le nom en grec veut dire aigre.

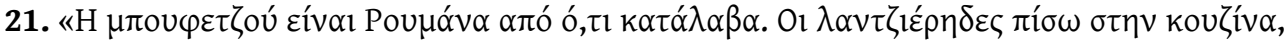

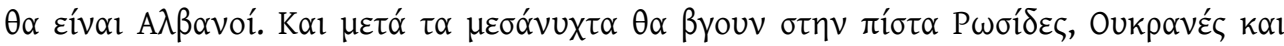

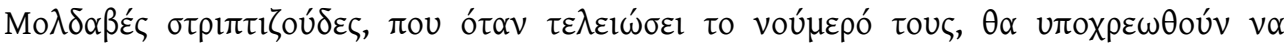

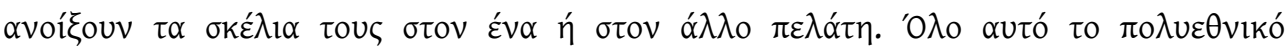

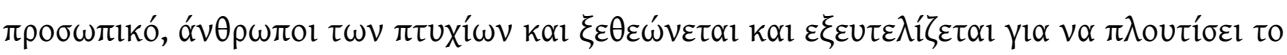

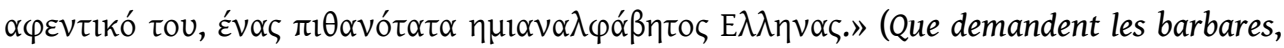
p. 149).

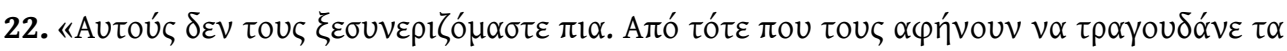

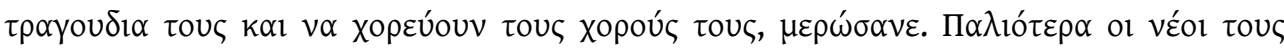

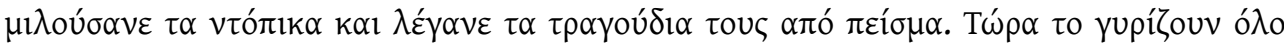

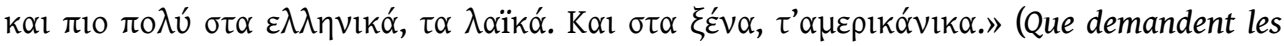
barbares, p. 23).

23. Sur la condamnation de l'État grec par la Cour européenne des droits de l'homme au sujet du respect des droits des minorités, voir les résultats de la mission de Gay McDougall, expert indépendant de l'ONU. Cette mission consistait à évaluer en Grèce, l'implémentation des dispositions prévues dans la Déclaration des droits des personnes appartenant à des minorités nationales, ethniques, religieuses ou linguistiques. Le rapport rédigé suite à cette mission est disponible sur l'adresse http://www.refworld.org/docid/49b7b2e52.html [consulté le 10 mars 2016].

24. Selon la définition de la nation par Ernest Renan.

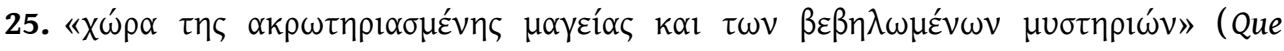
demandent les barbares, p. 31).

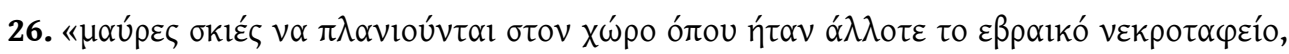

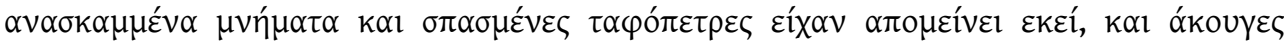

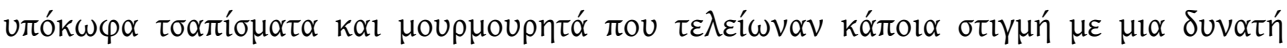

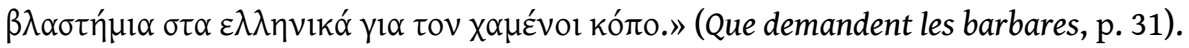

27. Sur l'histoire de la ville de Salonique pendant le $\mathrm{xIX}^{\mathrm{e}}$ et le $\mathrm{xx}^{\mathrm{e}}$ siècle, voir Régis DARQUES, 2000, Salonique au $\mathrm{XX}^{e}$ siècle: de la ville ottomane à la métropole grecque, et Mark MAzower, 2004, Salonica, City of Ghosts: Christians, Muslims and Jews, 1430-1950.

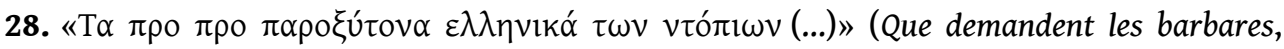
p. 31).

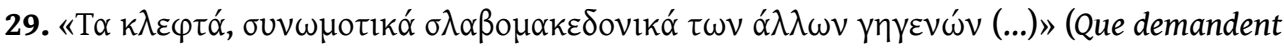
les barbares, p. 31). 


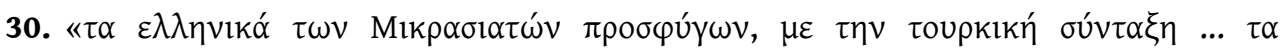

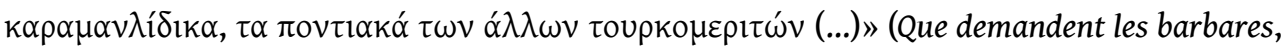
p. 31).

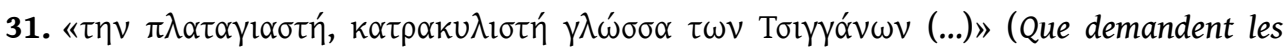
barbares, p. 31).

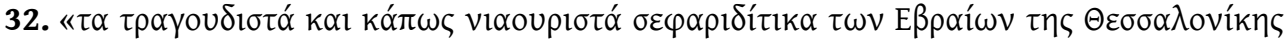
(...)» (Que demandent les barbares, p. 31).

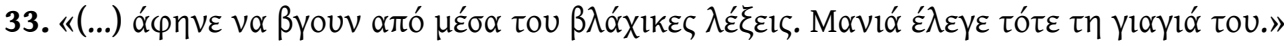
(Que demandent les barbares, p. 31).

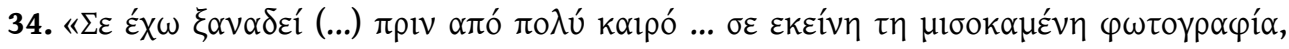

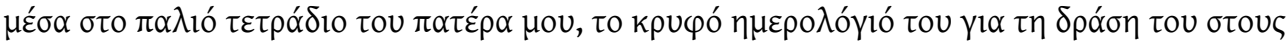

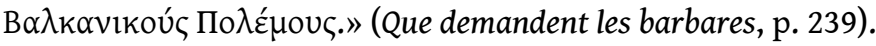

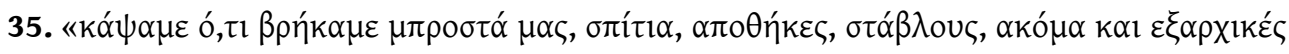

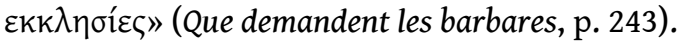

36. Il s'agit d'un rapport rédigé par les membres de la commission Carnegie qui ont remarqué que la propagande grecque avait excité l'opinion et préparé l'armée à combattre les Bulgares et à leur infliger un traitement inhumain. Entrés le 4 juillet à Kilkis, ville au nord de Salonique, dont la population était considérée comme favorable aux prétentions bulgares, les Grecs n'y laissèrent que des ruines. Voir George F. KENNAN, 1993, The Other Balkan Wars: a 1913 Carnegie Endowment Inquiry in Retrospect, pp.95-98.

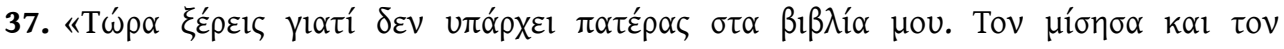

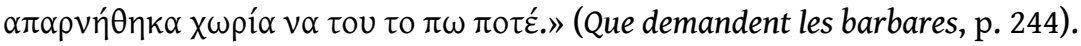

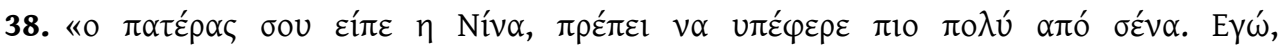

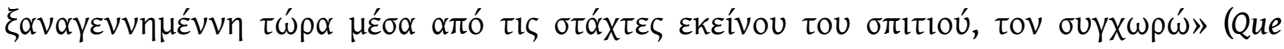
demandent les barbares, p. 244).

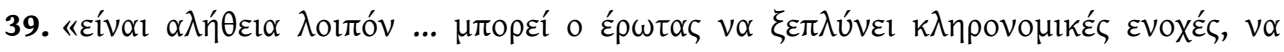

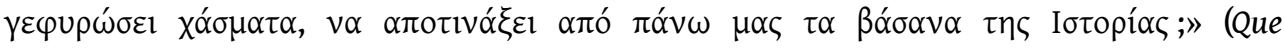
demandent les barbares, p. 244).

40. Voir Tásos KōsTópoulos, 2007, Guerre et nettoyage ethnique: l'aspect oublié d'une décennie d'expédition nationale, 1912-1922.

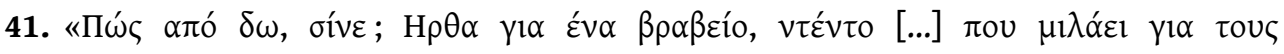

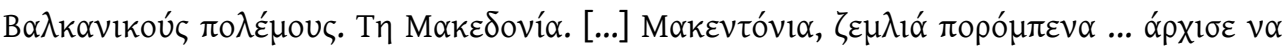

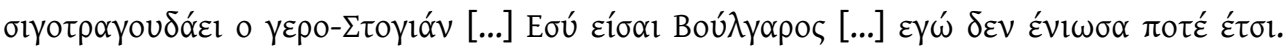

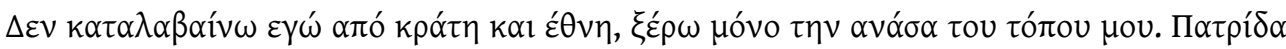

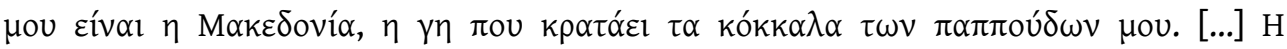

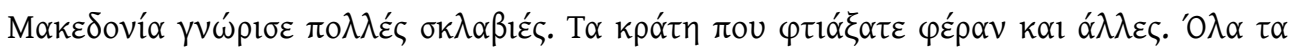
крátๆ.» (Que demandent les barbares, p. 51-52).

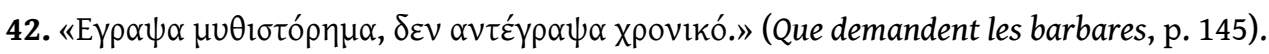

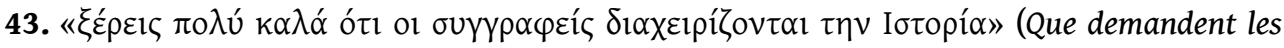
barbares, p. 147).

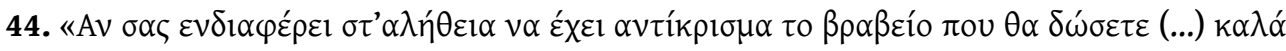

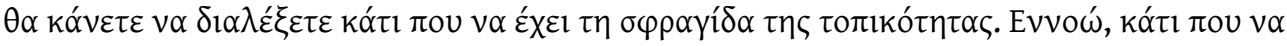

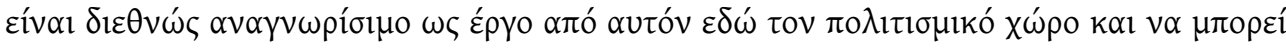

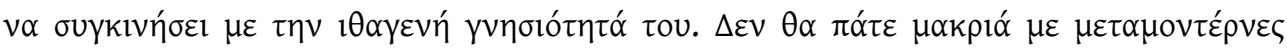




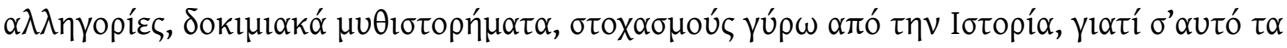

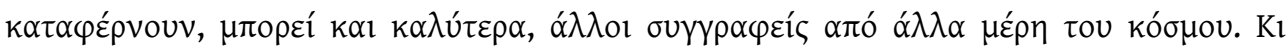

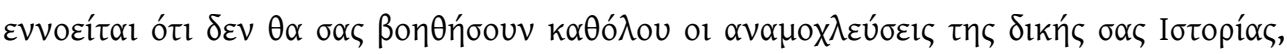

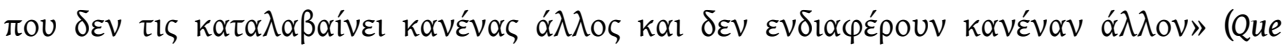
demandent les barbares, p. 184).

45. Dans sa qualité de critique littéraire, Koúrtovik avait déjà traité et soulevé cette question à propos de la difficulté de la littérature grecque moderne à acquérir une visibilité et une diffusion plus large dans l'espace-marché européen. Voir KоÚRTOVIK, 2005, le Hangover grec : essais.

46. Voir Arlette fARGE, «Penser et définir l'événement en histoire: approche des situations et des acteurs sociaux », p. 69-78.

47. Voir Hayden wHITE, 1973, Metahistory: the Historical Imagination in 19th Century Europe.

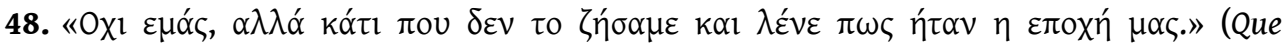
demandent les barbares, p. 210).

49. Voir Franklin R. ANKERSMIT, 1983, Narrative Logic: A Semantic Analysis of the Historian's Language. L'auteur, professeur d'histoire intellectuelle et de théorie de l'histoire à l'université de Groningen, reprend dans cet ouvrage certaines réflexions de Hayden White et tente d'esquisser la structure d'une philosophie de la narration. Comme le commente сісноска, Entre la nouvelle..., р. 98 : «ses réflexions sur la logique narrative de l'historiographie concernent non pas la possibilité d'existence d'un savoir sur le passé, mais plutôt la manière dont les outils linguistiques employés pour exprimer ce savoir déterminent sa nature ».

50. Pour cette typologie-classification des romans historiques, voir сісноскА, Entre la nouvelle..., p. 130.

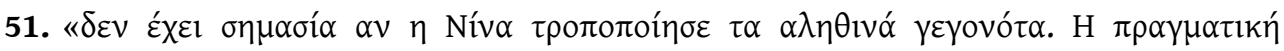

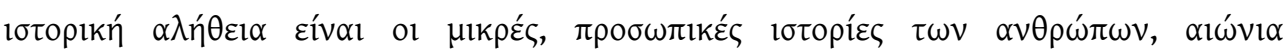

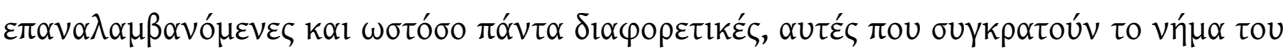
xpóvou» (Que demandent les barbares, p. 251).

\section{RÉSUMÉS}

Dimosthénis Koúrtovik situe la trame de son roman dans une ville fictive à l'occasion d'un concours littéraire balkanique. Ce concours devient le prétexte pour la confrontation entre trois lectures différentes d'un événement censé avoir eu lieu pendant la deuxième étape des guerres balkaniques de 1912-1913. Chaque narration à l'intérieur de ce roman, développe sa propre version des faits, basée sur l'exploitation de sources hypothétiques de nature variable, à la fois des journaux intimes, des correspondances ou des mémoires de famille. La construction et la structure du roman coïncident avec ce que Hayden White appelle des narratives concurrentes à l'égard d'événements historiques contentieux. Les débats focalisés sur l'historicité de l'intrigue et l'analyse d'éléments de la trame de ces différentes narrations en termes d'exactitude historique mettent en avant les relations compliquées tissées entre les romans traitant d'événements ou de 
périodes historiques et la réalité historique, notamment quand ces événements sont des objets de controverse entre les différents points de vue historiographiques. En dehors et peut-être en raison de ces réflexions théoriques au sujet de l'écriture de l'histoire et l'écriture de la fiction, ce roman et son auteur mettent en question le paradigme dominant nationaliste de lecture exclusiviste et nation-centrée d'événements historiques. Ce faisant, l'auteur revisite les guerres balkaniques, les ouvrant à une lecture pluraliste, tout en rendant possible une perception multiple de ce qui s'est passé comme cela a été transmis à travers des documents et des témoignages divers et variés.

Dimosthenis Kurtovic sets the plot of his novel in a fictive city during a Balkan literary contest. This contest allows the juxtaposition of three distinguished approaches of a supposedly real historical event occurring during the second phase of the Balkan Wars. Each novel develops its own version of what happened, based on various hypothetical sources, such as memoirs, correspondence or family memories. The novel's structure coincides with what Hayden White calls the competitive narratives regarding contentious historical events. The debates focusing on the historicity of the intrigue and the analysis of plot's elements in terms of historical accuracy, depicts the complicated relations woven between a novel which treats historical events or periods and the historical reality, particularly when these events are issues of controversy among different scholarship viewpoints. Besides or maybe thanks to these theoretical reflections between the writing of history and the writing of fiction, this novel and his author question as well the dominant nationalist paradigm of exclusivist and nationally-oriented lecture of historical events. In doing so, he revisits the Balkan Wars, opening them to a more pluralist lecture, allowing for a multiple perception of what happened as transmitted by different documents and various testimonies.

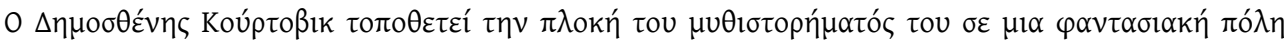

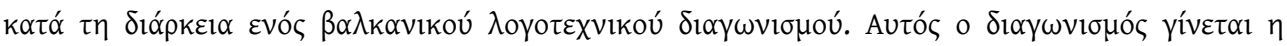

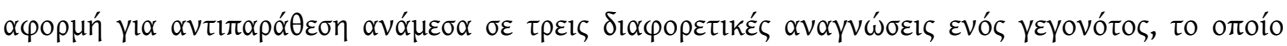

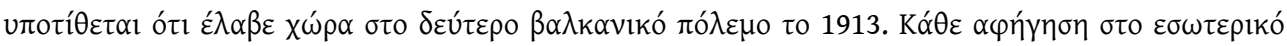

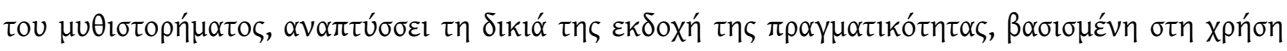

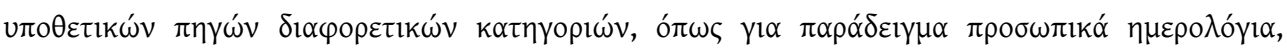

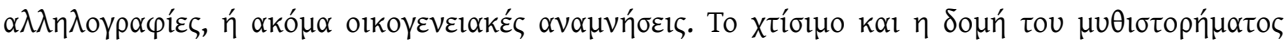

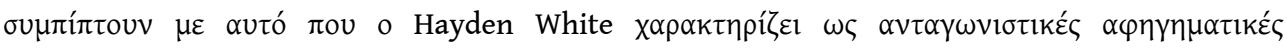

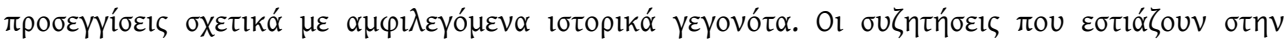

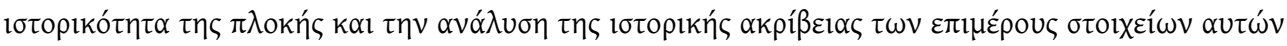

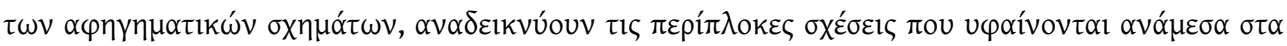

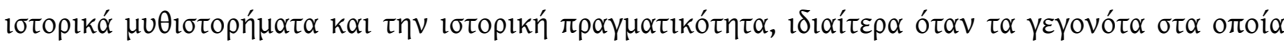

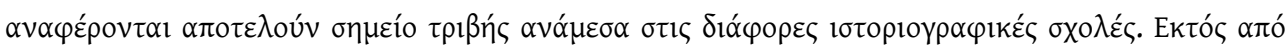

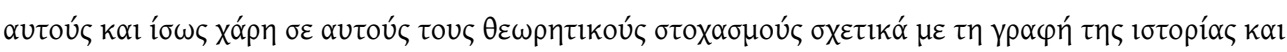

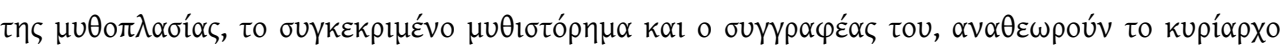

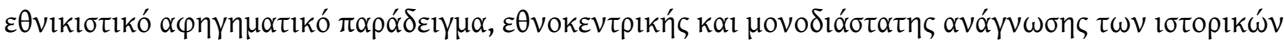

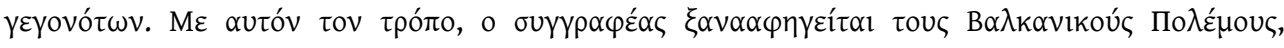

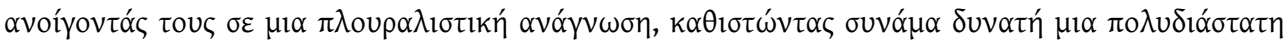

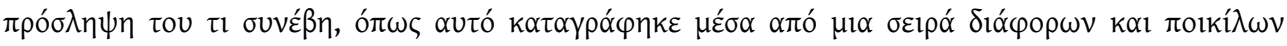

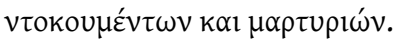




\section{INDEX}

Index géographique : Grèce, Macédoine

Mots-clés : question d'Orient, question d'Orient, nationalisme, nationalisme, racisme, racisme, xénophobie, xénophobie, Koúrtovik Dimosthénis (1948-), Koúrtovik Dimosthénis (1948-)

Keywords : Eastern Question, Nationalism, Racism, Xenophobia, Balkans, Greece, Macedonia, Twentieth century, Balkan Wars, Literature, History and literature

motsclesmk ИНТЕРТЕКСТУАЛНОСТА, НАЦИОНАЛИЗАМ, РАСИЗАМ, КСЕНОФОБИЈА, БАЛКАНОТ, ГРЦИЈА, МАКЕДОНИЈА, ДВАЕСЕТТИОТ ВЕК, БАЛКАНСКИТЕ ВОЈНИ, ЛИТЕРАТУРА ИСТОРИЈА И ЛИТЕРАТУРА

Thèmes : Littérature, Histoire et littérature motsclestr Doğu Sorunu, Milliyetçilik, Irkçılık, Yabancı düşmanlığı, Balkan, Yunanistan, Makedonya, Yirminci yüzyil, Balkan savaşları, Edebiyat, Tarih ve Edebiyat

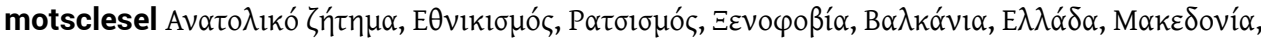

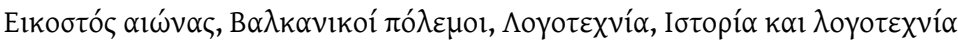

Index chronologique : vingtième siècle, guerres balkaniques (1912-1913)

\section{AUTEUR}

\section{NICOLAS PITSOS}

CREE, Inalco, Sorbonne Paris Cité 